\title{
Review Article \\ Initiating tumor banking for translational research: MD Anderson and Liverpool experience
}

\begin{abstract}
Mishra A, Pandey $A^{\star}$, Shaw $\mathbf{R}^{\star *}$
Department of Otolaryngology at King George's Medical University Lucknow, India and currently a Visiting Faculty and International UICC (American Cancer society) Fellow at the Department of Head and Neck Surgery, UT MD Anderson Cancer Center, Houston, USA, ${ }^{*}$ Department of Obstetrics and Gynecology, King George's Medical University Lucknow, India, **Department of Oral and Maxillofacial Surgery, University Hospital Aintree, Liver pool and currently a Visiting Scientist at the Department of Head and Neck Surgery, UT MD Anderson Cancer Center, Houston, USA
\end{abstract}

Correspondence to: Dr. Anupam Mishra, E-mail: anupampenn@yahoo.com

\begin{abstract}
The ultimate progress in the cancer diagnosis and therapy has only been possible with the ongoing translational research that is likely to play a very important role in future as well. Hence the importance of such translation from bedside to bench and visa versa cannot be over-emphasized. Accordingly it has become more important to collect tumor samples along with the clinical information in a systematic manner to perform a good basic science research in future. With a population of over a billion and a heavy burden of cancer, India has the 'biggest' potential to establish the 'largest' tumor bank across the globe. Establishing a tumor bank involves money and manpower that may not be feasible across most of the centers in India. Taking into the considering the model of tumor banking of the two leading institutions of the world (MD Anderson Cancer Center, USA and University Hospital Aintree, Liverpool UK), this article presents the salient tips for a center in India to get started with tumor banking with minimal investment. Furthermore a simplified form of ethical consent is presented for the centers to adapt unanimously.
\end{abstract}

Key words: Tissue-banking, tumor-banking

Tissue sample of tumors is the most important ratelimiting factor for carrying out any translational research. Such samples need to be properly processed, stored and carefully tagged with its clinical behavior. Hence a cancer research center needs to maintain a proper tissue bank from where serial stored samples along with the relevant clinical records can be obtained for a translational cancer research in future. Till date the concept of tissue banking in India is mainly concentrated in a few major cancer research institutes such as the Tata Memorial Hospital. Most of the cancer centers across the country do not have the concept of tumor banking. With over a billion population and an enormous cancer burden, such a concept would definitely unfold the enormous potential for translational cancer research in India. Considering the existing protocol of tumor banking at MD Anderson Cancer Center (USA) and University Hospital Aintree (Liverpool) we suggest some guidelines to initiate this concept in less well-equipped institutes of India. Ideally it has to be defined beforehand whether such a repository is a freestanding entity, virtual or a part of institution and moreover the standard operating procedures manual (SOPs) should be framed accordingly. Further discussion would highlight our views for starting this the concept as a freestanding entity with meager resources across the country.

\section{Anderson Cancer Center Protocol}

The head and neck tissue banking was started approximately 15 years ago at MD Anderson Cancer 
Center, Houston Texas and till date the bank has a total collection of approximately 25000 tissue specimen. This subsequently proved to be a model for Central MD Anderson Tissue Bank. The salient features of the protocol are as follows:

1. Ethics and consent process: Considering the ethics involved, a proper consent is essential before tissue procurement. A special tissue-procurement-nurse for this purpose visits the wards a day prior to surgery to obtain an open ended 'generic' consent or for specific study protocol.

2. Paperwork raised by the nurse is delivered to the staff of both operation theatre and pathology lab for pre-planning the specific medium requirements. In certain situations when preoperative consent is not available, the relevant attending staff is accordingly informed. The consent tissue can then be sought either 'retrospectively' or the tissue destroyed as the case may be.

3. As soon as the specimen is removed (biopsy or excision) in the operation theatre, the pathology lab is telephonically informed. The entire specimen is transported to the pathology lab as soon as possible.

4. The surgical specimen is examined and further 'cut' as per the size of the specimen. In case of large tumors $(>1 \mathrm{~cm})$ a small piece of tumor with normal margins is taken up for research that is subsequently coded and logged. Half is considered for optimum temperature for cutting (OTC) and other half is snap frozen. Research samples are compared to mirror image fixed specimens and thereby pathology checked following $\mathrm{H}$ and $\mathrm{E}$ examination. The remaining tissue is fixed and examined as normal. Following histopathological assessment, further specimens are retrieved from the paraffin blocks, such that half of the tissue represents the tumor and the other half surrounding 'normal' i.e., the invasive front, dysplasia, involved and uninvolved lymph nodes.

5. For small tumors ( $1 \mathrm{~cm}$ or less), no fresh tissue is retrieved in order to prevent damage to margins endangering useful diagnostic tissues. After histopathological assessment, a tumor sample and a normal sample is taken from the fixed specimen.

6. Hence for each major tissue excision, the following are available for research: (a) Snap frozen tumor $(>1 \mathrm{~cm})$; (b) Snap frozen normal margin (if $>$ $1 \mathrm{~cm})$; (c) OTC frozen tumor $(>1 \mathrm{~cm})$; (d) OTC frozen normal margin (if $>1 \mathrm{~cm}$ ); (e) fixed tumor; (f) fixed close margin; (g) fixed normal margin; (h) fixed lymph node; (i) fixed dysplastic mucosa.

7. Importantly the time interval between arrival in the pathology lab and freezing is recorded considering its importance in receptor / RNA studies where morphological changes may be important.

8. For specific research protocols or clinical trials other procedures are carried out such as snap frozen in operation theatres itself. Ideally the tumor specimen should be frozen within 10 min of surgical resection in order to prevent RNA degradation. The ideal storage place is the liquid nitrogen cylinder. The protocol for human sample processing as carried out at MD Anderson is depicted in Table 1.

A comprehensive and dedicated database for each specimen is maintained through intranet and it is planned to link tissue database with clinical database in near future. In order to maintain such a tissue bank, MD Anderson has three regular staff members solely dedicated for this purpose. A research technician carries out coding, database entry processing and storage of specimens and is also trained to prepare DNA, RNA, tissue micro-arrays etc.

\section{Developing Tissue Banking in India}

A very important technical aspect of concern is the temperature for storing the tissue specimen. Many scientists believe that storing the tissues at lower temperatures helps in preserving the integrity of the biopsy specimen for long-term, ${ }^{[1,2]}$ however there is no general consensus on this. Even RNA studies have been performed on paraffin block tissues but the sensitivity decreases with increasing temperature of storage. Hence the more lower the storage-temperature, the better. Ideally the specimen should be preserved in large liquid nitrogen cylinder, which unfortunately may not be available in the majority of centers across the country. In most medical colleges the departments of pathology and surgery are located in different buildings and hence a significant delay occurs in tissue transportation. In such situations it seems ideal to maintain a deep freeze refrigerator for small tissue collections in the respective departments. Several options for storing the tissues in a deep freeze refrigerator as a less expensive methodology can be considered based on UKBTS guidelines. ${ }^{[3]}$ The biopsy tissue must be placed in an ambient temperature of $-20^{\circ} \mathrm{C}$ within $48 \mathrm{~h}$ of retrieval. This does not only enhance bacteriostasis but is known to facilitate longterm storage for six months. Alternatively if such storage can be maintained at $-40^{\circ} \mathrm{C}$ then long-term storage can be maintained for a period of three years. It is worth mentioning that as per UKBTS guidelines, ${ }^{[3]}$ the tissue if preserved in high concentration glycerol may be stored at $0^{\circ} \mathrm{C}$ to $-10^{\circ} \mathrm{C}$ for up to two years. Considering the above methods it may be possible to store the tissue in heavy glycerine at $-40^{\circ} \mathrm{C}$ for a very long time. It has been a convention to store tissue at 


\section{Table 1: Processing procedures to be carried out and recorded including the time of collection and the type of specimen received}

\section{A. Peripheral blood:}

1. Specimen is collected in a heparin tube (vacutainer green cap).

2. Record specimen identification on log sheets, assigning it to the next available bank location.

3. Layer peripheral blood over $3.0 \mathrm{ml}$ histopaque (Sigma) found in $4^{\circ} \mathrm{C}$ fridge and centrifuge (2500 RPM for $15 \mathrm{~min}$ ).

4. Pipette buffy coat with a pipet and add $2.5 \mathrm{ml}$ PBS and transfer to a labeled IOx 13-glass tube.

5. Centrifuge (1500 RPM for $3 \mathrm{~min})$.

6. Decant supernatant and wash with PBS.

7. Add $1.5 \mathrm{ml}$ of PBS to pellet and mix by pipetting up and down.

8. Transfer to a labeled $1.5 \mathrm{ml}$ eppendorf tube, centrifuge (8000 RPM for 3 mins) and remove supernatant.

9. Place label eppendorf tube in designated box location.

B. Serum:

1. Specimen is collected in a no additive tube (vacutainer red cap).

2. Record specimen identification on the log sheet, assigning it to the next available bank location.

3. Centrifuge specimen vacutainer red cap tube (2500 RPM for 15 mins).

4. Aspirate $1.5 \mathrm{ml}$ of yellow serum from top of tube and transfer to a labeled $1.5 \mathrm{ml}$ eppendorf tube.

5. Place tube in designated box location.

\section{Sputum:}

1. Collected specimen in a specimen cup (white lid container).

2. Pipette $1.5 \mathrm{ml}$ of sputum to a labeled eppendorf tube. This aliquot is designated (type sputum liquid -under comment $1.5 \mathrm{ml}$ ).

3. Aspirate another $1.5 \mathrm{ml}$ of sputum to a second eppendorf tube and centrifuge (8000 RPM for $3 \mathrm{~min}$ ).

4. Decant supernatant from second tube leaving pellet at bottom of tube. This aliquot is designated (type sputum pellet-under comment pellet).

5. Place both tubes in the designated box location. Send remainder specimen for cytopathology.

D. Saliva

1. Collected specimen in specimen cup (white lid container).

2. Record specimen identification on the log sheet, assigning it to the next available bank location.

3. Pipette $1.5 \mathrm{ml}$ of saliva to a labeled eppendorf tube.

4. Place tube in the designated box location.

\section{F. Buccal scraping:}

1. Collected specimen will come with a wire brush inside.

2. Record specimen identification on the log sheet, assigning it to the next available bank location.

3. Pipette $1.5 \mathrm{ml}$ of specimen from brush and transfer to a label eppendorf tube. This aliquot is designated (type buccal scraping liquid- $1.5 \mathrm{ml}$ ).

4. Pipette another $1.5 \mathrm{ml}$ of specimen to a second eppendorf tube and centrifuge (8000 RPM for $3 \mathrm{~min}$ ).

5. Decant supernatant from second tube leaving pellet at bottom of tube. This aliquot is designated (buccal scraping-pellet).

6. Place tube in the designated box location. 
less that $-80^{\circ} \mathrm{C}$ for long term, but considering the practicalities in India, this glycerol-preservation seems to be the best alternative in initiating the process. Such 'compromised tissues' may not be well suited for RNA studies as such. It is not infrequent to have frequent short-time power failures in the absence of uninterrupted power backup in our facility. To withstand this, we suggest the use of cryogel bags around the storage racks inside the freezer to maintain the desired temperature in a 10 degree celsius range as advocated $^{[4]}$ for a prolonged time. However in case of prolonged electrical failure the freezer should be backedup with a UPS / generator facility. Also adequate backup capacity for low-temperature units must be maintained in anticipation of possible equipment failure. It is desirable to have satisfactory temperature (less than 22 degree Celsius), lighting and sufficient space for air circulation at the place where mechanical freezers are installed. Adequate ventilation is critical in liquid-N2 repositories and where dry ice is used to ensure that sufficient oxygen levels are maintained.

The most important issue in tissue banking is freely given informed patient consent. Tissue banks of human specimens for research must adhere to all central / state government and local regulations. Research on human specimen involves no interaction with patient and the risks are primarily from loss of confidentiality. ${ }^{[4]}$ Accordingly the processes and procedures for storage of human specimens for research should be available for review by an IRB to assure that they are appropriate to protect human subjects. For prospective collection of human biological materials the disclosure should include details of procedures (drawing blood, buccal swab), risks / benefits involved (bruising at site, access to records by others, no prospect of direct benefit) and guarded 'right-to-withdraw' because active participation ends at the time of collection, while sample would remain but links would be destroyed and genetic information would exist in the databases. Consent can be for a specific research use or for future unspecified uses. If the use is unspecified, an IRB review of the research must be conducted to assure that the use is consistent with the original consent. ${ }^{[4]}$ An example of the customized consent form based on a project 'Cytogenetic analysis of oral squamous cell carcinoma'is depicted in Table 2. This has been adapted from the 'University Hospital Aintree, Liverpool' protocol, where such tumor banking has recently been initiated. Table 3 depicts a sample of the relevant information necessary to be conveyed to the patient before consenting. Ideally all patient consent forms must be updated annually to be in compliance with IRB regulations.

Another important aspect is maintenance of clinical records and linking it to the specimen. Confidentiality of clinical records needs to be maintained specially in terms of identity of the subject. However the HIPAA 'Privacy rule' 1996 establishes a category of health information referred to as protected health information (PHI) which may be disclosed only under certain conditions. ${ }^{[4]}$ This is with an aim to permit researchers to review PHI in medical records or elsewhere to prepare a research protocol. Such a de-identified data would exclude all the possible subject identifiers and would be released after a non-disclosure agreement by the researcher. The Records need to be retained for a minimum of 10 years after the date of distribution / expiration of sample. Ideally the electronic records should be backed-up regularly on a network or remote server or on a CD / floppy.

The continuance, success and long term utility of a tumor bank depends upon its quality assurance (QA) and intermittent quality control checks. The QA programme of a tissue bank involves planning, implementation, documentation, assessment and improvement to ensure that the processing or specimen / item is of a standard quality. Quality control check on the other hand involves technical activities to measure the attributes and performance of a certain process or item against defined quality standards. It is important for a quality assessment program to maximally adapt to current-good-manufacturing-practices (cGMP)-regulatory guidelines as per its limitations. This would include secured area with limited access, trained personnel for required procedure, provision of internal QA audits by external agents, maintenance of extensive paper trail for all materials and equipments, documentation of equipment maintenance procedures as well as for all events that fall outside SOPs. ${ }^{[4]}$ It is important to note that ISO9001 is another system standard created through the international organization for standardization (ISO). ISO is similar to cGMP, but is more recognizable in international settings. Hence this may not initially suit a small repository of a developing country. All the tumor banks must be subjected to regular audits for example a weekly check of freezer temperature logs and a more complete review on a monthly or quarterly basis that includes random checks of SOPs for expiration and possibly pulling and checking random sets of specimens against the inventory list.

The consideration of safety is of paramount importance for those persons dealing with human samples in tumor banks. Apart from the general safety measures against fire, electrical and physical hazards as applicable elsewhere, special precautions need to be undertaken to protect the employees against blood-borne pathogens 
Table 2: Consent form

Date:

Identifying Number:

'Cytogenetic analysis of oral squamous cell carcinoma'

I have read the attached information sheet on the use of samples for the above project and have been given a copy to keep. I have had the opportunity to ask questions about the project and understand why the research is being done and any foreseeable risks involved.

I understand how the samples of tissue and cells will be collected and that giving samples for this project is voluntary.

I understand that I am free to withdraw my approval for use of samples at any time without giving a reason and without my medical treatment or legal rights being affected.

I agree to give blood samples for this project.

YES/NO

I agree to give tissue and cell samples for this project.

YES/NO

...........

I agree that the sample and the information I give will be looked after and stored at the University Hospital and YES/NO maybe used for future molecular research projects on head and neck cancer with ethical committee approval.

I give permission for my medical and related records to be examined and information taken from them for confidential use without revealing my identity.

YES/NO

I understand that some of these projects may be carried out by researchers other than the current team, both within the University Hospital and elsewhere.

YES/NO

I understand that some of these projects may be carried out by researchers working for commercial organizations.

YES/NO

I confirm that I understand the purpose of this research study. I am aware that donation of samples is voluntary and I can ask for these samples to be destroyed at any time, without my medical care being affected.

YES/NO

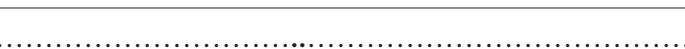

Name of Patient (Block Capitals)

Date

Signature

Consented by (Block Capitals)

Date

Signature

Please read carefully and sign each section. Adapted from University Hospital Aintree protocol

and diseases spread through skin-to-skin contact such as scabies and tuberculosis (specially in India). Vaccination against hepatitis should be encouraged. Accordingly every tumor bank should define the safety priorities and thereby develop a comprehensive safety programme in the light of national, state, regional and local regulations for health protection. It is preferable to establish a safety committee for planning, implementing and monitoring overall safety programme through a safety officer, which in turn collaborates with local area supervisors to establish safety-training-programmes. In the absence of such safety committee the legal responsibility of a hazard lies with the person designated or the overall in-charge of the tumor bank. Hence training for all the employees is mandatory in every domain (biohazards, chemical hazards, radiological hazards) and that too should be documented and updated each year. It is very important to note that all human specimens irrespective of state are to be treated as infectious since prions (C-J disease) may still be active with fixed tissues processed to paraffin blocks. Hence the use of apron coats, long pants, covered shoes, plastic gloves, glasses and masks protect the employee from biohazard as well as avoid chemical and thermal burns from both heat and cold (liquid $\mathrm{N}_{2}$ and dry ice). All chemicals should have material safety data sheets (MSDS) available for reference for employees who potentially will come into contact with these chemicals. MSDS are available from manufacturers. Storage of food / beverages should be strongly prohibited in laboratory areas and the same applies to mouth pipetting / suctioning. After handling potential hazardous material, hands and other exposed skin surfaces need to be thoroughly washed. A written emergency plan should be established to address the prevention, containment, cleanup and waste disposal in case of chemical spills. In general, some basic security systems such as alarms are desirable and a responsible person should take necessary action in a time frame to minimize the damage to collected material / specimen. 


\section{Table 3: Sample of information sheet}

University hospital cytogenetic analysis of oral squamous cell carcinoma 'INFORMATION SHEET'

The high incidence of head and neck cancer in <place> may be largely due to smoking habits. The aim of this project is to investigate the role of other factors in the causes of this disease. In order to achieve this, each subject will complete a short questionnaire, which will cover tobacco consumption and residential history and provide certain samples for analysis.

In this study we wish to identify people with tobacco smoking and chewing related changes in their mouth in order that we may be able to predict which individuals may have a greater risk of developing advanced oral lesions. In addition we wish to follow up individuals who have had treatment for oral lesions, in order that we can monitor these changes at a genetic level as well as routine clinical follow-up. By comparing the results of the tests on the scraped samples taken from the mouth from people without oral cancer, with those who develop oral cancer, we will be able to identify those people who have a very strong likelihood of developing oral cancer. Further research will be taking place to develop ways of intervening to prevent the disease from fully developing. Such intervention may take the form of chemoprevention (treatment with naturally occurring compounds), which can prevent the tumor growing.

We would like you to give a sample of blood and a scrape of cells from your mouth to help us with this research. In addition we would like to have a specimen of tissue, which maybe obtained during the tests you may have while you are in hospital or in the review clinics. We would only obtain these tissue specimens if they are surplus after making a diagnosis for you. When we receive samples we will use them to obtain chromosome (genetic material). The chromosomes from the blood samples and the oral tissue / scrapes will be used to look at the genetic changes that may lead to oral cancer as well examining the chromosomal aberrations people are born with which may increase their risk of oral cancer. The damaged chromosomes from cells from your mouth will allow us to predict the causative genes damaged by exposure to chemicals (for example those in tobacco smoke or industrial pollution). We hope that this information will help us to find new ways of detecting disease at a very early stage and then to find new effective treatments.

Our attempt is to find out what type of chromosomal pattern increases the risk of oral cancer. We do not know the answer to this question yet, so we will not be able to tell you the results of what we find. In other words, we are not using your chromosome sample to give you a 'genetic test' or a 'cancer test', but we hope we can use it to make such testing possible in the future. If a 'genetic test' should become available in the future - perhaps as a result of the research you are helping us with now - we would ask you if you would like to have this test and you would need to sign another consent form explaining the test. This is so we can make sure that you understand what the results would mean. We will not use any chromosomal sample given to this project for any purpose other than research and any results published will not relate to individuals or mention of any names.

If you agree to donate samples to us, the project co-ordinators will be responsible for looking after them. You must understand that if eventually this research leads to any new treatment or medical test you will not benefit financially.

The research we are carrying out is very new and changing all the time so we will store some of the chromosome, from both your blood and oral cells, for use in future research projects relating to oral cancer and other oral diseases. The aim of this research is to find new ways to detect oral cancer and related disease at a very early stage when treatment would be effective. For example we may use your oral cells to find new diagnostic tests. This might involve staining cells in new ways, which could be used to predict early disease. We may wish to share some of the samples or some of the information with other researchers who are working with us. If we do so the sample will be coded and no personal information about you will be disclosed.

It may be beneficial, in the future, to work with a commercial company to develop new medical tests or treatments. We may wish to share some of the samples or some of the information with other researchers working for such a company. If we do so the sample will be coded and no personal information about you will be disclosed.

Any additional studies will be approved by the relevant ethics committees and the project co-ordinators will be responsible for protecting your confidentiality.

Giving a sample for this research is voluntary and you may withdraw your approval for use of the sample at any time and request that your samples are destroyed.

If you are harmed by taking part in this research project, there are no special compensation arrangements. If you are harmed due to someone's negligence, then you may have grounds for a legal action but you may have to pay for it. Regardless of this, if you wish to complain about any aspect of the way you have been approached or treated during the course of this study, the University Hospital mechanisms should be available to you.

(Adapted from University Hospital Aintree protocol)

Electricity safety can be assured by proper grounding of electric equipments, using electrical base plugs in good condition, proper use of fuses and surge-protectors as well as ensuring that water sources (sinks) are away from electrical devices. Fire safety can be ensured by storing flammable agents in fire cabinets, purchasing refrigerators/ freezers that are noncombustible, use of different types of fire-extinguisher-cylinders and strictly prohibiting smoking. Carefully analyzing the work environment and correctly applying ergonomics 
thereafter can enhance physical safety. This would also significantly reduce the visual and musculoskeletal discomfort. Considering the overall security it is important to limit access of unauthorized personnel in the workplace.

For initiating tumor banking following 'materialprotocol' may be adapted:

1. The large postoperative surgical specimen soon after excision should be incised with a fresh disposable blade into four pieces with margins. The first piece should be stored in formalin. The second piece should be further subdivided into multiple pieces (1$2 \mathrm{~mm}$ ) and each sub-piece should be stored separately both in normal saline and heavy glycerine in the deep freezer. The idea is to prevent multiple cycles of freezing and thawing while retrieving a small piece for study. During retrieval of the specimens, the plastic cryovials should be thawed at room temperature, while glass vials should be thawed overnight in a refrigerator to prevent cracking. The third piece should be stored as a paraffin block after histopathological examination and the fourth piece should be reserved for any special research protocols e.g., microbiological assessment for fungal elements etc.

2. For a small specimen, one half should be sent for histopathology and the other half retained separately in small aliquots both in normal saline and glycerine in the deep freezer.

3. A very small tissue specimen such as that obtained through biopsy should be sent as a whole to pathology lab for paraffin block. With a few millimeters of specimen, tissue banking may be limited to obtaining a paraffin block only but after establishing the diagnosis.

4. The other patient samples such as heparinised whole blood, plasma, urine, saliva etc can be stored preferably in deep freezer or alternatively as per the desired protocol. Table 1 depicts the processing procedures adapted from MD Anderson Cancer Center. It is important to rinse the pipette-tips with $10 \%$ bleach solution before discarding.

The 'linking' of clinical records with the stored specimen through the use of simple Microsoft access software seems to be most appropriate initially. This preliminary level of tumor banking is applicable to most of the medical colleges across India. The training of post-graduate residents for tissue collection, processing, storage and retrieval would be much easier and definitely desirable since with limited resources, it would be difficult to employ separate staff for the tumor bank. This would further minimize the hazards associated with the program. In places where storage in liquid nitrogen cylinders is feasible, it is important to note that the vapor-phase avoids safety hazards inherent in the liquid-phase storage. It may not be possible to barcode the aliquots and hence appropriate coding for example including the patient's initial, date of collection or some alternate method should be adapted. It is important to use proper labels / stickers and ink to last for at least 10 years.

Certain recently built cancer centers across the country have an advantage that the pathology facility exists in close vicinity to the operating theatres in the same hospital premises. This facilitates the tissue specimen from all the departments to be processed at a single place only, thus reducing the expenses incurred for manpower and equipment, that otherwise need to be maintained at the respective departments. However if the geographic distance is substantial, the postoperative tissue specimen can be temporarily stored for transportation in either small liquid-nitrogen cylinders or a tight thermacol box with frozen cryogel bags. Alternatively if the geographical distance is not much the fresh specimen may be transported in a sterile container on wet ice from surgery to pathology / repository. This still facilitates the concept of tissue processing at only one specific place, thus reducing the additional costs. To adapt the MD Anderson model of tissue banking it is essential to have the following:

1. Dedicated manpower / employees for this purpose only. The involvement of post-graduate residents would overcome this limitation in most of the centers across the country.

2. A good maintenance of database linking the tissue specific details with clinical details. Defined protocols for filling up the specific details need to be developed in this regard.

The concept of tumor banking at various levels of complexity needs to be incorporated amongst the teaching medical colleges across the country. This would be an asset for future translational studies in terms of tissue access that otherwise may not be feasible (to obtain) from a distant place (say for example virtual tumor bank EORTC data center) due to cost restraints in most of the centers in India. It may not be feasible to carry out all types of translational research in the beginning but after adapting increasing complexities of tumor banking, the stored biological specimens may become sensitive for high quality basic research.

\section{Acknowledgment}

The work done (main author: AM) has been supported by a UICC American Cancer Society International Fellowship for Beginning Investigators. The author would like to further 
acknowledge Dr. Adel El-Naggar MD, Professor, Department of Pathology and Dr. GL Clayman MD Professor, Department of Head and Neck Surgery, UT MD Anderson Cancer Center, Houston for their expert inputs.

\section{References}

1. Karlsson JO, Toner M. Long term storage of tissues by cryopreservation: Critical issues. Biomaterials 1996;17:243-56.

2. Eiseman E, Bloom G, Brower J, Clancy N, Olmstead SS. Case studies of existing human tissue repositories 2003. [Last accessed on 2006 Feb 1]. Available from: http://www.rand.org/ pubs/monographs/2004/rand_mg 120.sum.pdf.

3. UKBTS Guidelines, Tissue retrieval and processing (chapter 22),
Guidelines for the blood transfusion services in the United Kingdom. [Last accessed on 2006 Feb 1]. Available from: http:/ /www.transfusionguidelines.org.uk/uk_guidelines / ukbts6_213.html.

4. Best practices for repositories I: Collection, storage and retrieval of human biological materials for research (ISBR Best Practice Guidelines). Cell Preservation Technology. 2005; 3(1):5-48. [Last accessed on 2007 Feb 15]. Available from: http://www.isber.org/ pubs/bestpractices.pdf.

Source of Support: UICC American Cancer Society International Fellowship for Beginning Investigators, Conflict of Interest: None declared.

\section{FORM IV}

Statement about ownership and other particulars about newspaper (Indian Journal of Cance", '? ie published in the first issue every year after the last day of February

1. Place of publication

2. Periodicity of its publication

3. Printer's Name Nationality

Address

4. Publisher's Name Nationality Address

5. Editor's Name Nationality Address
: Mumbai

: Quarterly (January, April, July and October)

Medknow Publications Pvt. Ltd.

Indian

A-108/109, Kanara Business Center,

Off Link Rd, Ghatkopar (E),

Mumbai - 400075 India

Phone: 91-22-f/j49 1818/1816,

Fax: 91-22-รぇ7? 1817

Medknow Pıiplications Pvt. Ltd.

Indian

A- 0r/109, Kanara Business Center,

Uff Link Rd, Ghatkopar (E),

Mumbai - 400075, India

Phone: 91-22-6649 1818/1816,

Fax: 91-22-6649 1817

: Jagdeesh N. Kulkarni

Indian

INDIAN CANCER SOCIETY

Lady Ratan Tata Medical and Research Centre,

M. Karve Road, Cooperage, Bombay - 400021, India.

Phone: 91-22-2202 9941/2202 9942,

Fax: 91-22-2287 2745,

Web: www.indiancancersociety.org

6. Names and addresses of individuals who own the newspaper and partners or shareholders holding More than one percent of the total capital.

: Indian Cancer Society

I, Jagdeesh N. Kulkarni, hereby declare that the particulars given above are true to the best of my knowledge and belief. 